\title{
Computer Aided Formal Design of Swarm Robotics Algorithms *
}

\author{
Thibaut Balabonski ${ }^{1}$, Pierre Courtieu ${ }^{2}$, Robin Pelle ${ }^{1}$, Lionel Rieg ${ }^{3}$, Sébastien Tixeuil ${ }^{4}$, and \\ Xavier Urbain ${ }^{5}$ \\ ${ }^{1}$ Université Paris-Saclay, CNRS, LMF \\ ${ }^{2}$ Cédric, Conservatoire des Artts et Métiers, Paris \\ ${ }^{3}$ VERIMAG, UMR 5160, Grenoble INP, Univ. Grenoble Alpes \\ ${ }^{4}$ Sorbonne University, CNRS, LIP6 \\ ${ }^{5}$ Université Claude Bernard Lyon 1, LIRIS UMR5205
}

\begin{abstract}
Previous works on formally studying mobile robotic swarms consider necessary and sufficient system hypotheses enabling to solve theoretical benchmark problems (geometric pattern formation, gathering, scattering, etc.).

We argue that formal methods can also help in the early stage of mobile robotic swarms protocol design, to obtain protocols that are correct-by-design, even for problems arising from real-world use cases, not previously studied theoretically.

Our position is supported by a concrete case study. Starting from a real-world case scenario, we jointly design the formal problem specification, a family of protocols that are able to solve the problem, and their corresponding proof of correctness, all expressed with the same formal framework. The concrete framework we use for our development is the PACTOLE library based on the COQ proof assistant.
\end{abstract}

\section{Introduction}

\subsection{Context}

Swarm robotics envisions groups of mobile robots self-organizing and cooperating toward the resolution of common objectives, such as patrolling, exploring and mapping disaster areas, constructing ad hoc mobile communication infrastructures to enable communication with rescue teams, etc. As several of those applications are life-critical, the correctness of the deployed protocols becomes of paramount importance. In turn, correctness reasoning about autonomous moving and computing entities that collaborate to achieve a global objective in a setting where unpredictable hazards may occur is complex and error prone. A first step into more formal reasoning is to use a sound mathematical model.

Suzuki \& Yamashita [28] introduced such a mathematical model describing the behaviour of robots in this context. The model is targeted at swarms of very weak robots evolving in harsh environments. At its core, the model simply commands individual robots to repetitively observe their environment before computing a path of actions to pursue and acting on it, usually by moving to a specific location. Three different levels of synchronization have been commonly considered. The fully-synchronous (FSYNC) case [28] ensures each phase of each cycle is performed simultaneously by all robots. The semi-synchronous (SSYNC) case [28] considers that time is discretized into rounds, and that in each round an arbitrary yet non-empty subset of the robots are active. Finally, the asynchronous (ASYNC) case [19] allows arbitrary delays among the Look, Compute and Move phases, and the movement itself may take an arbitrary amount of time. The Look-Compute-Move model received a considerable amount of attention from the Distributed Computing community, ${ }_{1}^{1}$ yielding a large variety of submodels induced by refined system assumptions. Such submodels were typically used to assess the solvability of a certain

\footnotetext{
*This work was partially supported by Project SAPPORO of the French National Research Agency (ANR) under the reference 2019-CE250005-1.

${ }^{1}$ Yamashita received the "Prize for Innovation in Distributed Computing" for his seminal work on this model.
} 
task assuming certain system hypotheses. As such, the Distributed Computing literature about mobile robots so far can be seen as computability-oriented.

Alas, the various submodels make it extremely tedious to check whether a particular property of a robot protocol holds in a particular setting. Furthermore, these variants do not behave well regarding proof reusability: checking that a property holding in a given setting also holds in another setting that is not strictly contained in the former often amounts to developing a completely new proof, regardless of the proof arguments similarity. This constitutes a major issue when one investigates the correctness of new solutions or implementations of existing protocols to be used in more realistic execution models. This problem is specially acute because of the great diversity of subtly different models: one may be tempted to simply hand-wave their way around the issue by declaring that the proof in this model is "obviously" also valid in this very close model, even more so as even a careful examination may not always find the most subtle errors. Last but not least, protocols are typically written in an informal high level language: assessing whether they conform to a particular model setting is particularly cumbersome, and may lead to hard to find mismatches. As a result, sustained research efforts were made in the last decade to use formal methods in the context of mobile robotic swarms.

\subsection{Related works}

Formal methods encompass a long-lasting path of research that is meant to overcome errors of human origin. Perhaps the most well known instance in the Distributed Computing community is the Temporal Logic of Actions and its companion tools TLA/TLA+ $[13,24]$. Though very expressive, TLA is designed for the shared memory and message passing contexts, thus not perfectly suited to studying mobile robotic swarms. Model-checking and its powerful automation proved useful to find bugs in existing literature $[6,17,18]$, and to assess formally published algorithms $[6,16]$, in a simpler setting where robots evolve in a discrete space where the number of possible positions is finite. Automatic program synthesis (for the problem of perpetual exclusive exploration in a ring-shaped discrete space) is due to Bonnet et al. [8], and can be used to obtain automatically algorithms that are "correct-by-design". The approach was refined by Millet et al. [25] for the problem of gathering in a discrete ring network. However, those approaches are limited to instances with few robots. Generalizing them to an arbitrary number of robots with similar models is doubtful as Sangnier et al. [27] proved that safety and reachability problems are undecidable in the parameterized case. Another limitation of the above approaches is that they only consider cases where mobile robots evolve in a discrete space (i.e., graph). This limitation is due to the model used, that closely matches the original execution model by Suzuki and Yamashita [28]. As a computer can only model a finite set of locations, a continuous 2D Euclidean space cannot be expressed in this model. Défago et al. [14] used a more abstract model to model-check rendez-vous algorithms in a continuous 2D Euclidean space, however, their model is highly specific to rendez-vous and thus is not as versatile as one could hope, hinting at a more general and systematic technique.

The approach on which we focus in this work is formal proof, that is proof development mechanically certified by a proof assistant. Mechanical proof assistants are proof management systems where a user can express data, programs, theorems and proofs. In sharp contrast with automated provers (like model-checkers), they are mostly interactive, and thus require some kind of expertise from their users. Sceptical proof assistants provide an additional guarantee by checking mechanically the soundness of a proof after it has been interactively developed. Formal proof allows for more genericity as this approach is not limited to particular instances of algorithms. During the last twenty years, the use of tool-assisted verification has extended to the validation of distributed processes, in contexts such as process algebras [7,20], symmetric interconnection networks [21], message passing settings [23], and self-stabilization [1,15], etc. The main approach for mechanized proof dedicated to swarms of mobile entities is so far the PACTOLE ${ }^{2}$ framework. Initiated in 2010, The PACTOLE framework enabled the use of high-order logic to certify impossibility results, as well as soundness of protocol, for swarms of autonomous mobile robots. To certify results and to guarantee the soundness of theorems, the proof assistant it uses is CoQ. Briefly, CoQ is a Curry-Howard-based interactive proof assistant that enjoys a trustworthy kernel. Its base language is a very expressive $\lambda$-calculus, the Calculus of Inductive Constructions [10], where datatypes, objects, algorithms, theorems and proofs can be expressed in a unified way, as terms. The syntax is close to that of an ML-like programming language, and a proof development consists in trying to build, interactively and using tactics, a $\lambda$-term, the type of which corresponds to the theorem to be proven (Curry-Howard style). The small kernel of COQ simply type-checks $\lambda$-terms to ensure soundness. Most importantly : a theorem or a lemma can only be saved/defined in the system if it comes with its type-checked proof. Designed for mobile entities, and

\footnotetext{
${ }^{2}$ https://pactole.liris.cnrs.fr
} 
making the most of COQ's assets, PACTOLE allows for working on a given protocol to establish and certify its correctness $[4,12]$, as well as for quantifying over all protocol so as to prove impossibility results [2,5,11], with an unspecified number of robots, possibly including a proportion of Byzantine faults, in continuous or discrete spaces. FSYNC/SSYNC and ASYNC modes are all supported, and the framework is expressive enough to state and certify formally results as theoretical as comparisons between demons or models [3].

\subsection{Our Contribution}

Taking some perspective over aforementioned works mixing formal methods and swarm robotics, one can only notice that the computability-centric approach of the Suzuki and Yamashita model yielded a concentration of efforts towards few benchmark problems that are theoretically interesting (one can get impossibility results or correctness certification) but of little practical relevance, such as perpetual or terminating exploration of a ringshaped graph, and gathering or concentrating all robots at a particular location.

On the other side, relevant practical problems, such as constructing ad hoc mobile communication infrastructures to enable communication with rescue teams, remain untouched using a formal approach. Yet, their correctness is crucial, and possibly life-critical, so it should be assessed formally and mechanically verified. Overall, for those practical problems, the question is not really to characterize which system hypotheses enable problem solvability, but rather how to design a provably correct solution using hypotheses that correspond to real devices.

This paper is the first step in this direction. In more details, we start from a real-life application scenario to jointly design (i) its formal specification, (ii) a family of protocols that are able to solve the problem, and (iii) their corresponding proof of correctness, all expressed with the same formal framework, PACTOLE. In this process, we illustrate how formal methods and PACTOLE in particular could be used to derive protocols that are correct-by-design before they are deployed to actual devices.

The developments for COQ v8.12 described in this work are available at https://pactole.liris.cnrs. fr/pub/connection_8.12.tgz

\section{Search and Rescue, the life line maintainance problem}

One of the most advertised applications of robotic swarms is the Search and Rescue situation: a devastated zone has to be explored to bring assistance to survivors. A particular scenario in that context is the "life line" one where, static communication infrastructures having been destroyed, a robotic swarm establishes a dynamic network between a moving search team and the rescue station, by sending mobile transmitters relays.

The same problematic is faced in the less dramatic context of the pursuit of a hornet with drones whose sensors and transmitters are of limited range [26].

In such situations :

- A mobile target follows an unpredictable path and must always be in sight.

- Mobile robots with limited sensors/transmitters track and follow the target.

- To maintain contact with the base station which might be lost due to range limits, some of the robots act as relays.

The link between the base and the target, the so-called life line, must never be broken. More precisely: the existence of such a life line should be ensured at each and every point in the execution.

As practical applications may be critical, with lives at stake, it is imperative for that invariant to hold, and one needs formal guarantees about it.

\subsection{Space}

Focusing on the search and rescue scenario, we have drones flying and monitoring a rescue team on the ground. Though the most natural space would be the 3D space, it is enough to consider that the mobile robots are moving on a continuous plane, at a fixed altitude. Thus, we can choose the space to be the 2D Euclidean plane. ${ }^{3}$

In this space, there is a point called the base from which robots are launched. The second defining object is the companion which moves on the plane and follows the rescue team, regardless of other robots; it has to be connected to the base, one way or another.

\footnotetext{
${ }^{3}$ This incidentally will allow us to use vertical movement as a way to remove robots from the protocol, see Section 4.2 .3 .
} 


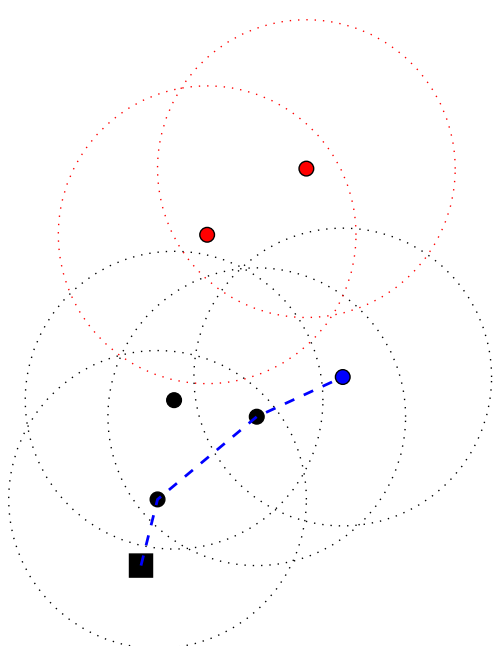

(a)

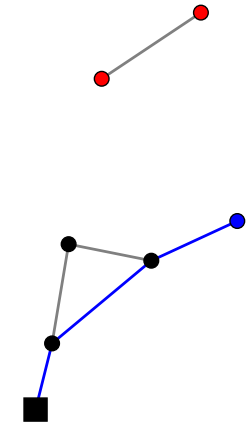

(b)

Figure 1: Let us consider in (a) a configuration of the system where $\mathbf{\square}$ is the base, $\bullet$ is the companion, and where visibility range are denoted as dotted circles. In this situation, the two robots o cannot detect the others. Nonetheless there is a chain of visibility between the base and the companion, denoted as a dashed blue path. The corresponding visibility graph is in (b), with a suitable path we ask for in blue.

\subsection{Basic robot characteristics and sensors}

To avoid the problem being trivially solvable, we require the sensors and transmitters to have a limited range. Hence we grant robots the ability to see neighbouring robots up to some finite range $D_{\max }$. Indeed, infinite range would make the whole protocol moot as a single robot located at the base would be able to see the target.

Note that we merge vision and transmission capabilities. As a matter of fact relays that do not see others would probably be lost, and the point of a relay is to have neighbours to transmit to.

There is no need to consider multiplicities, as the protocol has to avoid collision: two launched robots will never be at the same location (provided this property holds in the starting configuration).

Similarly, to avoid any trivial counter-example involving a straight line fleeing of the companion following the search team, we constrain the speed of drones to a certain $D$ denoting the bound on the distance that can be travelled within one cycle. Also, we only consider executions where the number of available relays is sufficient.

\subsection{Mode of synchronicity, movements, and initial configuration}

We may assume the execution to be fully synchronous, with a short time span for each cycle. As pointed out in Section 1.3, our goal is not to find minimal synchrony assumptions that allow problem solvability, but rather make sensible and realistic assumptions that permit deployment with actual devices. When robots are homogeneous, they operate at a similar pace, and the fully synchronous model is a sensible choice. Furthermore, keeping the time span short makes the assumption close to what continuous time practitioners envision [9, 22].

For the same reason, we also consider movements to be rigid, that is robots do not start a new cycle before they have reached their computed destination. Since robots always select as a target another robot within the visibility radius, movements are always of limited length.

The fact that the rescue team departs from the base allows us to define a notion of valid initial configuration. Trying to solve the problem if the rescue team is already out of reach would make no sense in that context.

\subsection{Informal specification of the problem}

As it is critical to keep the rescue team connected to the base, we want a formal guarantee that there is always a chain of robots going from the base to the target, such that robots along this chain can relay communication.

Given a configuration of the system (see e.g. Figure 1), we say that two robots are connected if they are located at most $D_{\max }$ apart, that is within visibility range. We formalize this idea by a visibility graph, where nodes are 
robots and edges relate connected robots. Thus, having a chain of connected robots going from the base to the companion can be expressed as having a path from the base to the companion in the visibility graph.

A second objective of our protocol is to avoid collision for obvious reasons, that is, to make sure that no two distinct robots are ever at the same location.

Overall, we end up with:

- A list of hypotheses characterizing our context and the environment, on space, sensors, synchronicity...

- An invariant that, provided there are enough relays to be sent, has to hold at each point of any execution starting from a valid configuration: there is a path in the graph of visibility and there is no collision.

\section{A Brief Overview of the PaCtole Framework}

One of the aims of PACTOLE is to stay as simple and as close as possible to the definitions of the robotic swarm community.

Thus, a state of the overall system, a configuration is defined as the collection of the states of all robots, conveniently combined into a map from robot names to robot states:

Definition configuration $:=$ ident $\rightarrow$ state.

A robot state can be anything (to accurately describe reality) but must at least contains its location, accessible through a function get_location : state $\rightarrow$ location, where the type location denotes the space where robots evolve.

An execution is an infinite sequence of configurations:

Definition execution := Stream.t configuration.

Executions are usually built by executing a protocol (called a robogram) against an environment, represented as a demon, that is, an infinite sequence of decisions called demonic actions.

Definition demon := Stream.t demonic_action.

The robogram represents the Compute part of the Look-Compute-Move cycle. It takes the observation of the robot as input and outputs the action that the robot should perform.

Definition robogram := observation $\rightarrow$ action.

This observation denotes a degraded version of the configuration centred on the observing robot, depending on its sensors. It is a parameter of the model and its computation from a (local) configuration is performed by an obs_from_config function, which hides the information unavailable to robots and takes as input the configuration and the state of the observing robot. This function is specified by a logical formula obs_is_ok relating any configuration to its possible observation from any robot state.

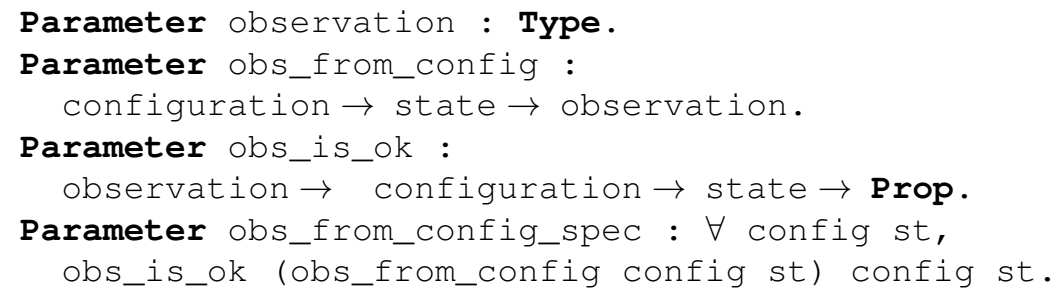

To represent the fact that robots observe from a personal point of view, they have their own frame of reference that need not be consistent in time or with other robots (other orientation, other scale, other origin, etc.). This frame of reference allows to create a local configuration (by opposition to the point of view of the demon denoted as global) from which the observation is computed, it depends on the underlying space and it is picked by the demon.

In such an execution, the robogram corresponds to one Look-Compute-Move cycle and the demonic action to the reaction of the environment. Their interaction is described by a function round so that the resulting execution is simply repeatedly calling this function with the robogram, the demon and the starting configuration.

The round function is the heart of the model, implementing the Look-Compute-Move cycle and computing the configuration obtained after one round. Note that this function is the same for all variants, FSYNC/SSYNC/ASYNC synchronization, all spaces, all sensors, etc. This is done in the following consecutive steps for each robot name id: 
1. If the robot $i d$ is not activated, its state may undergo some change by the inactive function to represent an ongoing action or the effect of the environment.

2. If $i d$ is a byzantine robot, it is relocated by the demonic action da.

3. Use the local frame of reference provided by da to compute the local configuration.

4. Transform this local configuration into an observation.

5. Apply the robogram on this observation.

6. If moves are flexible, compute the new position of id using information given by da.

7. Convert the new position from the local frame to the global one.

The full round function is:

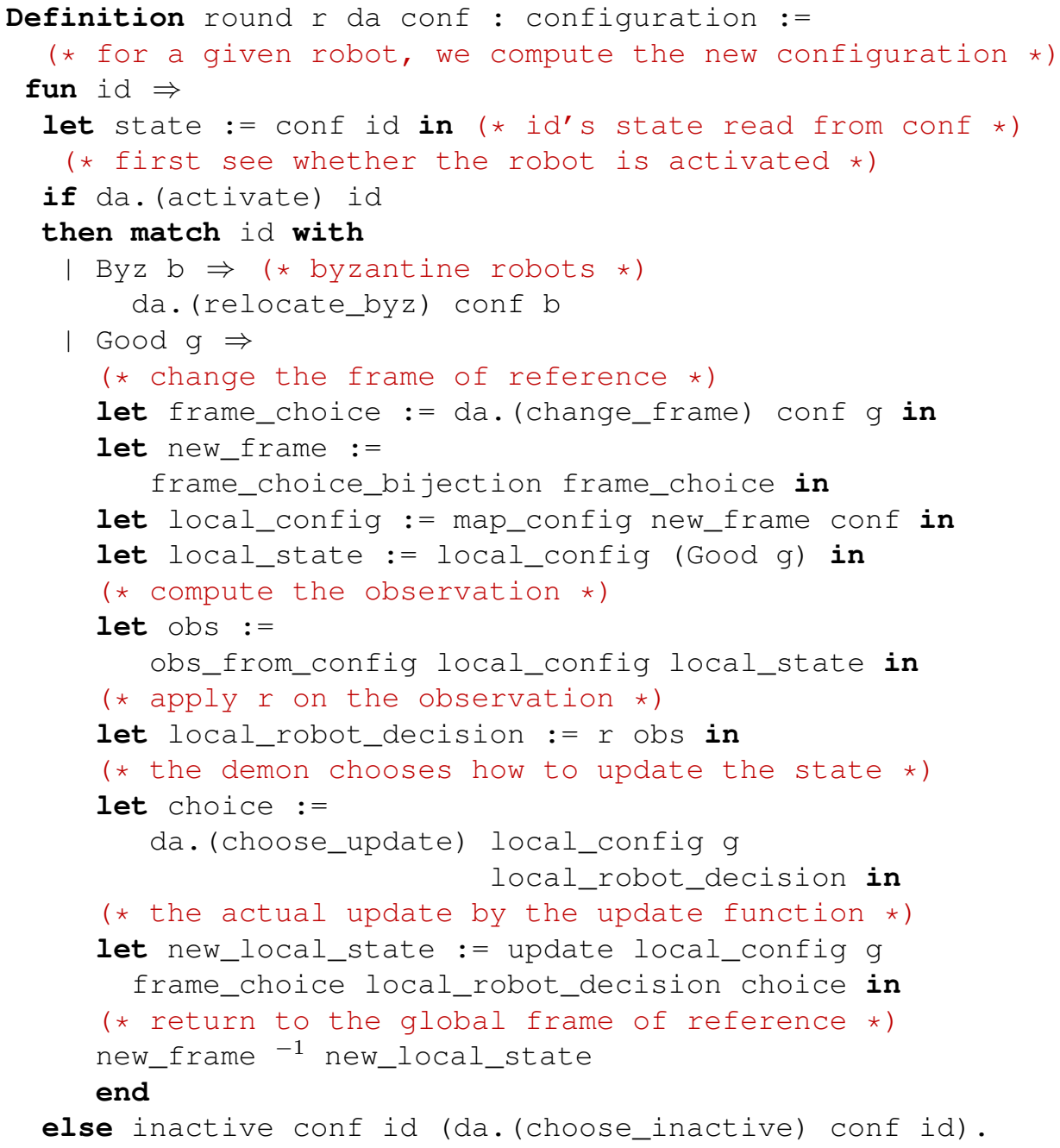

\section{Co-Designing Specifications, Solutions, and Proofs within the Formal Framework}

The first step is to instantiate in the formal framework the assumptions listed in Section 2, as well as the property that we want to be holding throughout a relevant execution.

Those specifications are "refined" until a set of constraints on robots and protocol candidates is formally proven sufficient. Ultimately, our family of protocols will be given by an abstract protocol parameterized by several functions that will be specified enough for the correctness proof to hold. 
The final specifications are thus designed together with a solution and its proof.

For the sake of readability we use $\mathrm{cf} x$ as a shorthand for $\mathrm{Cf}$ (Good $\mathrm{x}$ ), i.e. the position of a (non-Byzantine) robot named $\mathrm{x}$ in a configuration $\mathrm{cf} .{ }^{4}$

\subsection{Specifications}

The parameters of the problem are the number $n$ of robots, the visibility radius $D_{\max }$ of robots and the maximum distance $D$ they can travel in a round.

Parameter $\mathrm{n}$ : nat.

Parameters D Dmax : R.

Using the number $n$ of robots, we define canonical names to be able to refer to them:

Instance Robot_Names : Names := Robots $\mathrm{n} 0$.

The 0 represents the absence of Byzantine failures.

The space is the 2D Euclidean space, already predefined in PACTOLE:

Instance Loc : Location := make_Location R2.

Initially, a robot's state contains only its location, and its observation consists of the set of inhabited locations within its vision range:

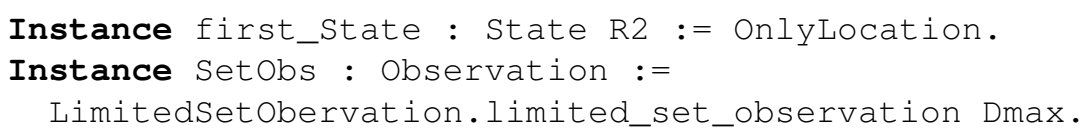

The changes of frame of reference allowing to switch between global and local observation (and vice-versa) are similarities (that is, moving the origin around and changing the orientation, chirality, and scale):

Instance first_Frame : frame_choice (similarity location)

$:=$ FrameChoicesimilarity.

The FSYNC setting is reflected in an hypothesis made over demonic actions, by requiring property FSYNC_da to hold (as will be seen the final statement of the invariant).

Movements are defined as rigid.

Instance setting_is_rigid : RigidSetting.

As a consequence of our FSYNC and rigid setting with no Byzantine failure, some parameters of the PACTOLE framework are not used and can be set to arbitrary values, namely what happens to a robot whenever it is inactive, how the demon interferes with a robot movement, what happens to robot suffering Byzantine failures, etc. Essentially, one can skip the first two steps of the round function described in Section 3 and focus on the LookCompute-Move cycle.

Context \{inactive_choice_ila : inactive_choice bool\}.

Instance demon_upd : update_choice unit := Nochoice.

We say of a robot participating to the task that it is launched (by the base station) and alive. (These notions will be formally motivated and introduced during the design of the protocol, respectively in Sections 4.2.2 and 4.2.3.) The main goal of any candidate protocol is to maintain the following properties at any moment during the execution:

1. There is no collision between robots, i.e. any two distinct alive robots not at base do not share the same location:

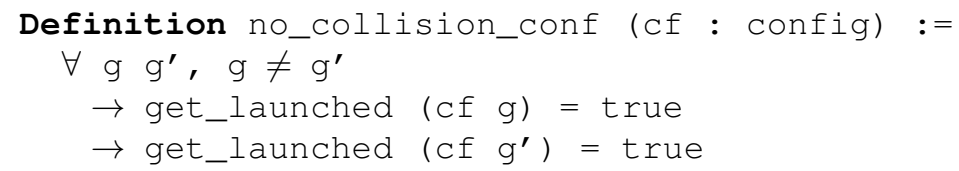

\footnotetext{
${ }^{4}$ For the sake of clarity, some notations may slightly differ between the actual code and this section, and some irrelevant technical overhead may have been pruned.
} 


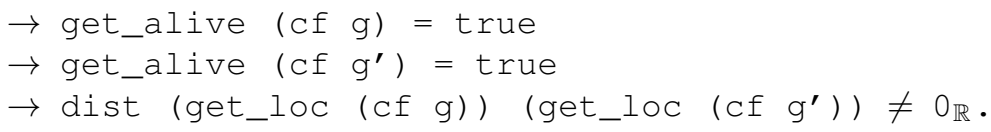

2. There is a sequence of connected robots from the base to the companion (numbered 0 , always alive). In other words: for any robot alive (we shall see that there is always some robot alive on base) either it is the companion or it has a visible, active, launched neighbour with a smaller id.

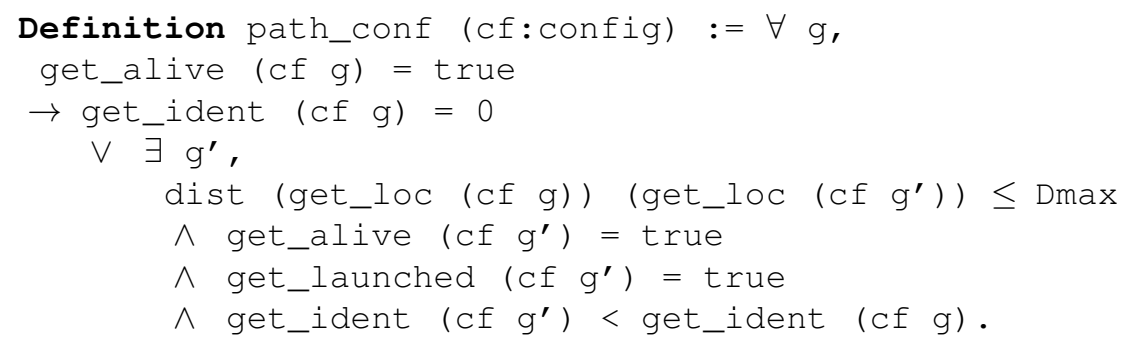

Note that this definition implies the existence of a connection from the base to the companion only if there is a robot alive (launched or not) close to the base, i.e. the set of robots waiting to be launched never gets exhausted. The fact that this property always holds during the considered execution is stated as the premise exists_at_based below.

The preservation of this invariant is expressed as

Definition NoCollAndPath e :=

Stream.forever (Stream.instant

(fun cf $\Rightarrow$ no_collision_conf cf $\wedge$ path_conf cf)) e.

Being assumed that the invariant holds in the initial configuration config_init The final lemma will state that the invariant holds forever along any execution of the (abstract and parametric) protocol rbg_ila, provided that the set of robots alive at base never gets empty.

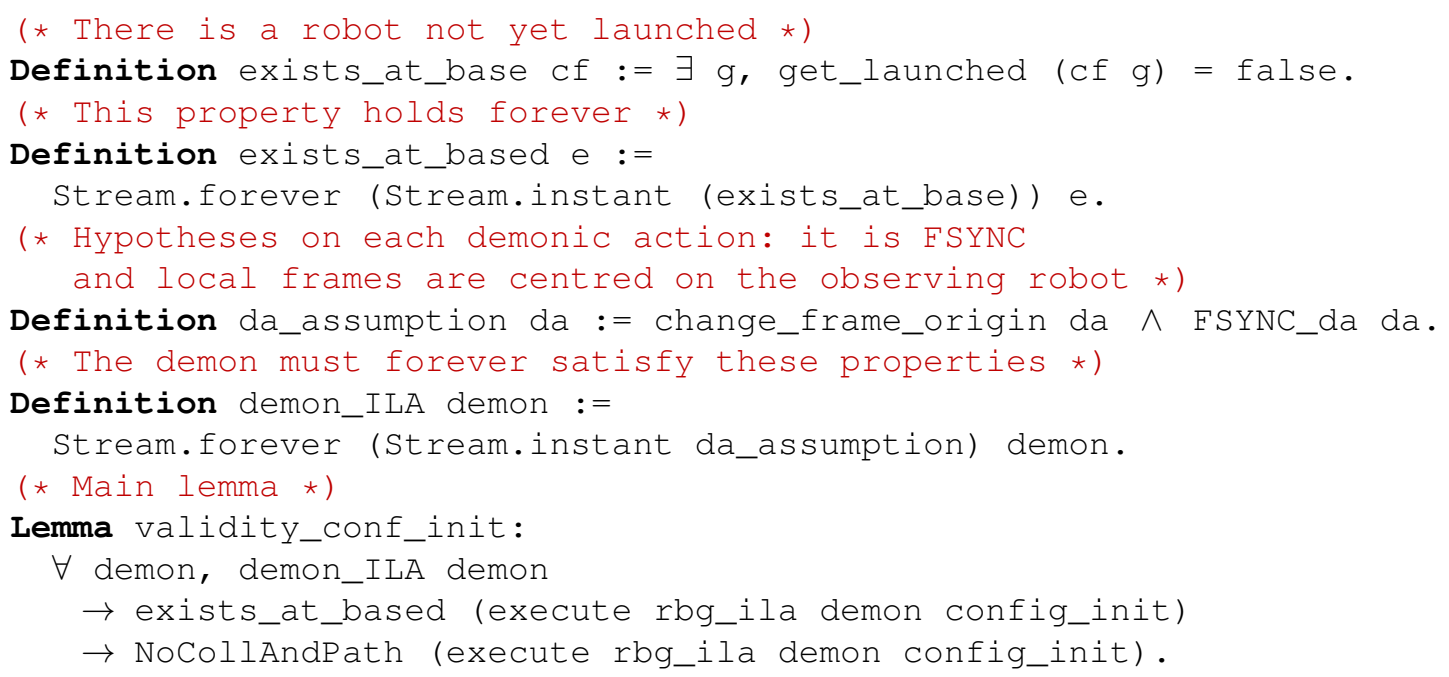

We show in the remainder of this work that the aforementioned lemma can in fact be formally proven for any rbg_ila fulfilling some sufficient conditions that we discovered in the next section. A concrete suitable protocol is outlined in Section 4.4.

\subsection{The March towards a Family of Solutions}

We describe in the following how we refine this initial formal model according to the issues we ran into and the hypotheses (whether necessary or by design) we find helpful along the protocol and proof co-development. We update the formal instantiation accordingly. For the sake of clarity, we use the following notation: the successive re-definitions of the parameters are indexed with the refinement step (e.g. State $e_{2}$ is second refinement of the initial default robot state $\left.S t a t_{0}\right)$. We drop that numbering when we reach the final version of the parameters. 


\subsubsection{Robot path needs an orientation}

Problem: When robots see neither the base nor the companion, for instance when they only see their two neighbours on the life line, they have no way of knowing in which direction the base lies and in which direction the companion lies.

This information is important since the behaviour of relay robots is not symmetric: they should follow the companion as the base is responsible for launching new relay robots whenever necessary. Therefore relay robots need a way to know in which direction lies the companion.

Solution: We provide visible identification and a strict ordering between those robot identifiers. To this goal we use a unique positive integer per robot, and assume that robots will be launched in ascending order. This way, the direction of the companion is given by smaller identifiers. The definition of a state is updated accordingly.

Note that the newly introduced identifiers are visible information and are not to be confused with Names of robots (Section 4.1), which are constructs internal to the formal framework.

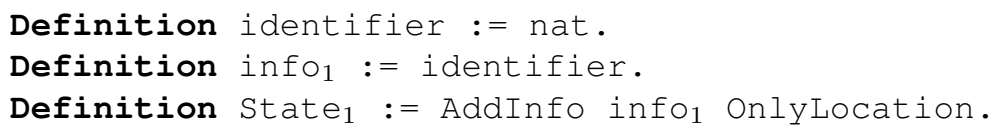

The companion will be given number 0 , and at any moment if a robot is launched then all other robots already launched have a smaller id.

\subsubsection{Distinguishing robots waiting at base}

Problem: What if a robot is located at the base but is already launched? Should this configuration be indistinguishable from the one where it is not yet launched? Obviously not, as collisions may occur in the former but not in the latter.

Solution: The state of a robot should contain a boolean indicating if the robot is "launched" or not. The state should be updated accordingly.

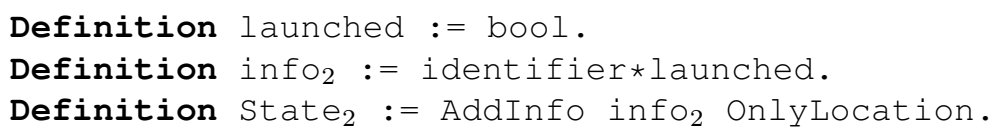

\subsubsection{Robots may have to withdraw from the system}

Problem: When the companion comes closer to the base, the connection line shrinks making the connecting robots possibly unable to stay out of collision risk. Therefore a useful feature of a candidate protocol would be the ability to withdraw from the line.

Solution: We assume available any arbitrary procedure for robot removal, and we add a boolean alive to the robot state, making sure that dead (that is, withdrawn) robots are not taken into consideration by alive robots. ${ }^{5}$ For simplicity and separation of concern, we make dead robots not even observable by the other robots by adding new constraints to the specification of the observation.

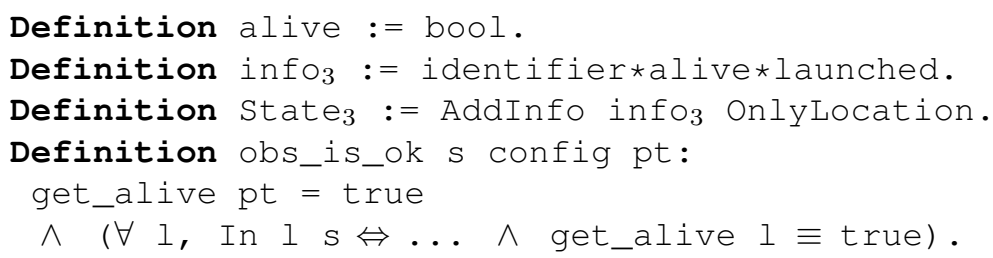

${ }^{5}$ For instance, we may make them change altitude making sure they no longer represent a collision risk. 


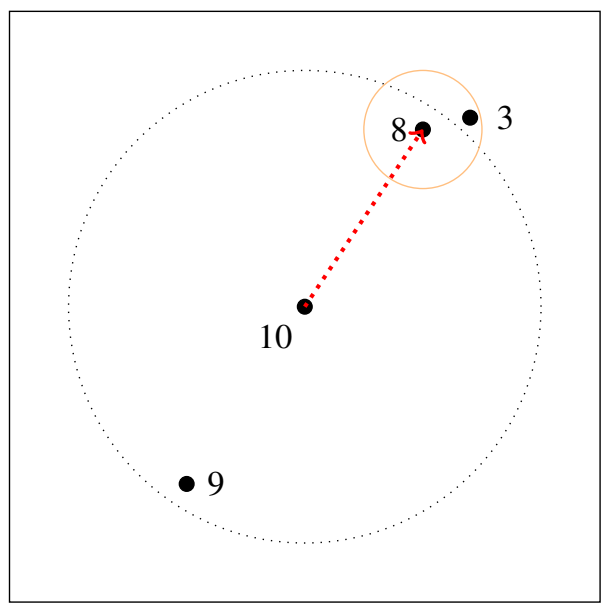

(a)

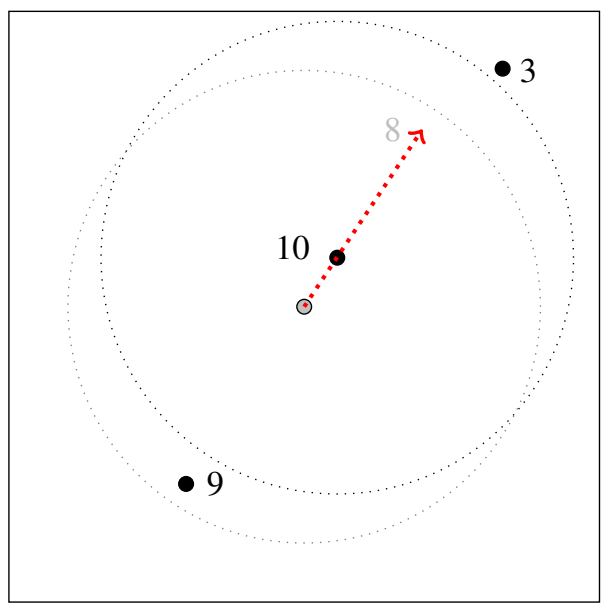

(b)

Figure 2: In situation (a), robot 8 will withdraw as it is too close to 3 . Robot 10 can see 8 and 9,3 is out of sight, and it may choose 8 as its target. Situation (b) shows what can happen in this case: 10 moves towards 8 (its previous position is shown in grey), which disappears. With 3 moving further, 10's connection with any suitable robot is lost.

\subsubsection{Pruning the space of candidate solutions}

Since robots are ordered, and the problem essentially revolves around following some robot of lower identifier, we choose to consider protocols where any given robot makes its decisions depending only on the robots of lower identifier. While this choice is not an answer to any particular problem, it reduces drastically the design space we explore. Once we make this assumption, several formerly open choices become closed. In particular, no robot of small identifier will ever take into account robots of higher identifier nor try to avoid collision with them. That means avoiding collisions and, if necessary, withdrawing, will always be the responsibility of the robots with higher identifiers.

\subsubsection{Robots need to warn neighbours before withdrawing}

Problem: A robot too close to others shall disappear, but there are cases where a robot immediately behind it may move too far and break the link, precisely because it tried to avoid that collision, even if that collision is now impossible due to the robot removal. This situation is illustrated on figure 2 . We decide to give robots the ability to warn neighbours about the possibility that it may withdraw in the next round.

Solution: We add a light to the robots capabilities. The light of a robot is either on or off. Any robot that sees another robot sees its light. Light on means the robot might be about to withdraw and should not be followed anymore, see Figure 3.

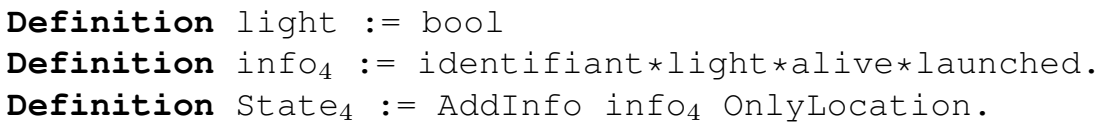

\subsubsection{A robot cannot predict the moves of other robots}

Problem: Any robot must ensure it avoids collision with other robots and keep in range the neighbour it chooses to follow. Since robots do not know their neighbours views, they cannot predict the moves other robots are about to make. Thus any robot must choose its move so that collisions are avoided and connection with the followed neighbour is preserved for any possible simultaneous move of the other robots.

Solution: Since we assume no robot can travel a distance greater than a constant $D$ in one cycle, we take all decisions with a $D$ margin. This entails defining several zones around a robot using this distance $D$, summed up 


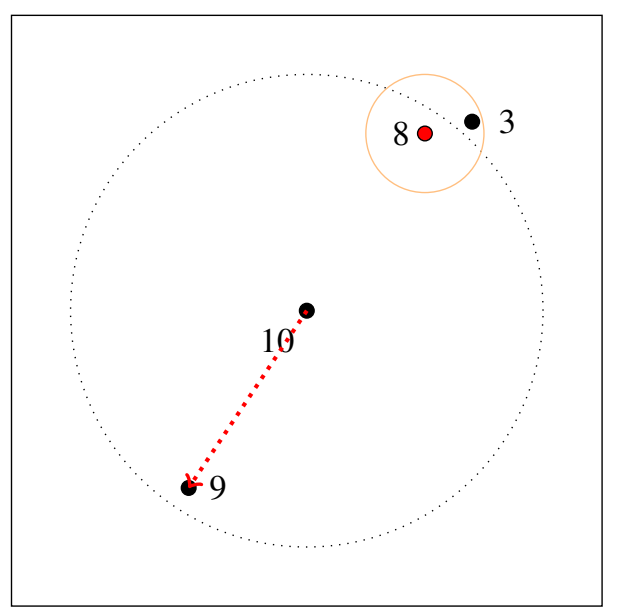

(c)

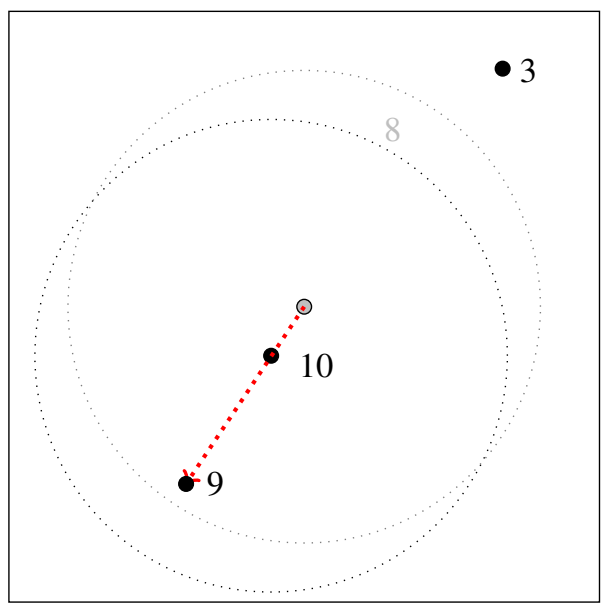

(d)

Figure 3: Situation (c) is similar to Figure 2 (a) as 8 will withdraw but now it switches its light on (here in red). As 10 knows that 8 is likely to disappear, it chooses 9 as target, thus keeping a connection with a robot of lower identifier (situation (d)).

in Figure 4.

- A neighbour at distance less than $D$ from the range limit, that is a neighbour at a distance comprised between $D_{\max }-D$ and $D_{\max }$ might become out of range after its move. If the followed neighbour is in this particular range, a move is necessary to ensure connection. This defines the Pursuit Zone. The distance of pursuit is then defined as $D_{p}=D_{\max }-D$.

- A neighbour at distance less than $D$ might provoke a collision. Since cycles are atomic we do not model how robots move inside a cycle and we can never ensure that robots with crossing trajectories will not collide. Thus the distance $D$ defines a Collision Zone. When two robots are at a distance less than $D$, either they have to agree on moving apart, or one has to withdraw.

- A neighbour that is not in the Collision Zone, but is still at a distance less than $2 D$ might enter the Collision Zone after its move. This range defines the Danger Zone, and letting another robot enter this zone means risking an imminent collision.

The range between distances $2 D$ and $D_{\max }-D$ gives no particular constraint: neighbours in this Relay Zone are neither too close nor too far.

\subsubsection{Robot vision radius must be large enough w.r.t. to robots speed}

Problem: A robot may not see far enough to connect without risk. An exemple of this situation is given on Figure 5 where too long a wait to avoid collision risks at launch time leads to a connection break.

Solution: We put a defensive safe limit for launching distance at $D_{\max }-4 D$, small enough to avoid losing robots in the example. It must also be large enough to allow for safe launch, at least $3 D$. A bound follows on $D_{\max }$ that must be strictly greater that $7 D$.

\subsection{A family of solutions}

At this point, we have gathered a few conditions that appear to solve the problems we foresee, and should help in maintaining the invariant. As we formalized them in the PACTOLE framework, we may now prove formally that indeed any protocol fulfilling these conditions is a solution to our connection problem. We define that way a family of protocols satisfying our needs.

Let us first recap the description of this family of solutions before moving to the proof of its correctness. 


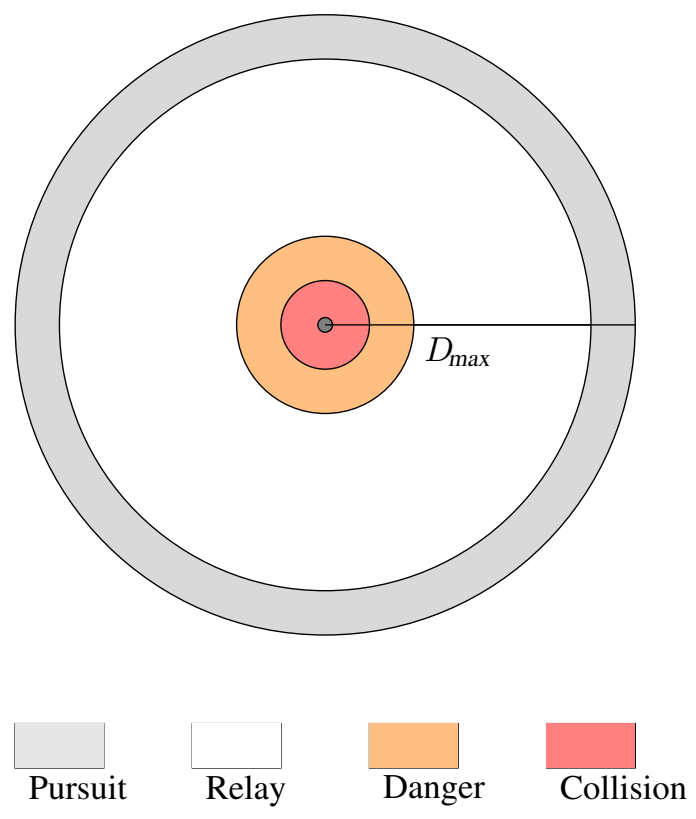

Figure 4: The visible surroundings of a robot can be divided into several zones that characterize the situations it may encounter: need to follow a robot (Pursuit zone, width $D$ ), high risk of immediate collision (Collision zone, radius $D$ ) or possibly at the next round (Danger zone, width $D$ ), and finally just transmission (Relay).

\section{Description of the family of solutions}

We describe the family of solutions to the problem by exhibiting a generic protocol rbg_fnc parameterized by three auxiliary functions.

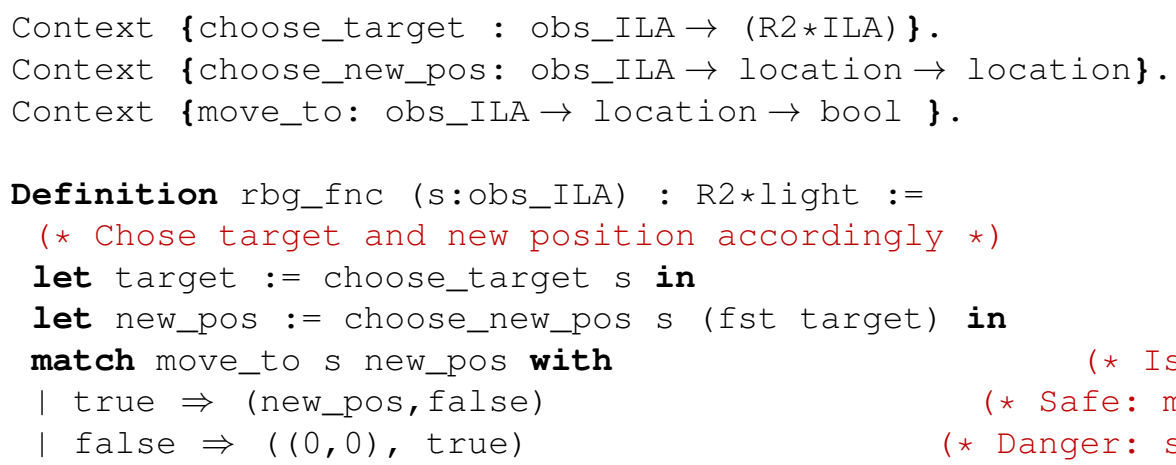

The role of choose_target is to select among the visible other robots the one that should be followed, i.e. the one we should maintain connection with on the next round. The role of choose_new_pos is to decide, given the target computed by choose_target, where the robot should move to ensure connection to it. Finally, the role of move_to is to decide whether it is dangerous to move to the selected new position. If that is the case, then the protocol decides not to move and warns neighbours that it may withdraw soon by turning on its light (and therefore should preferably not be selected as a target by another robot on the next round).

Our claim is that any three functions verifying the specifications described below will make the protocol achieve our goal. In the following section we give an example of a working instance, thus ensuring that this family is not empty.

The first hypothesis specifies the behaviour of the choose_target function. ${ }^{6}$ It expresses that its output must:

- be in range, that is be within the input (an observation) to choose_target;

\footnotetext{
${ }^{6}$ In the actual code, each of these five properties is split into its own independent statement for greater flexibility.
} 

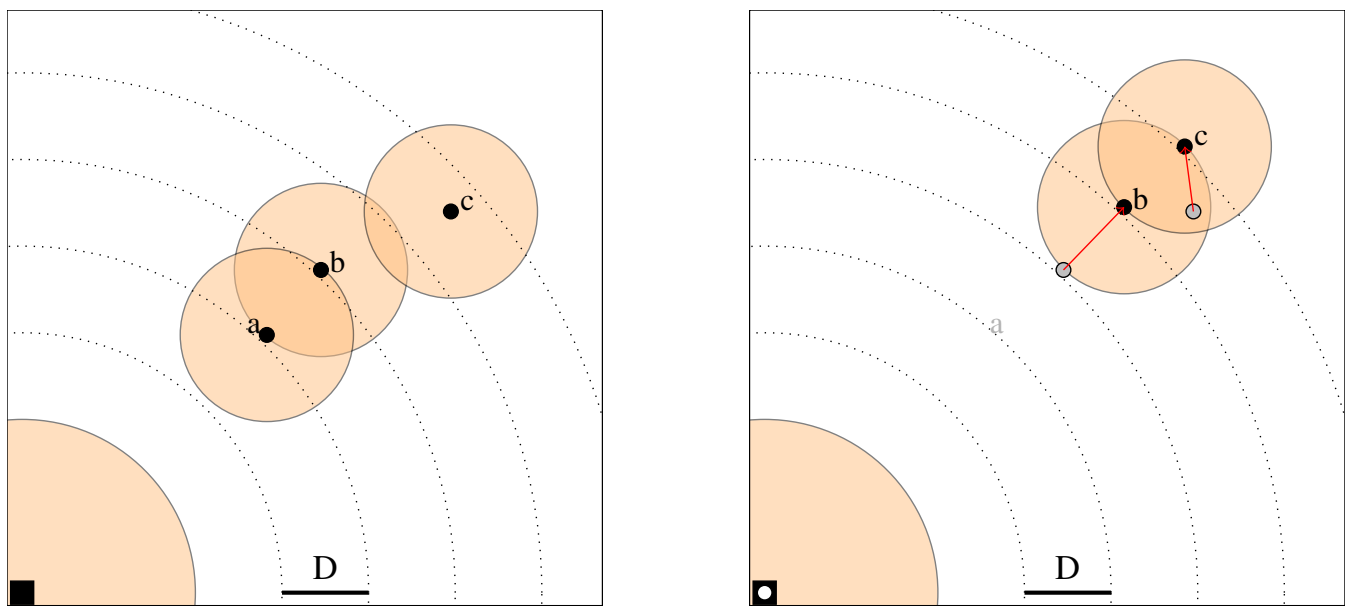

Figure 5: To avoid elimination at launch, the base waits for a robot a to be at more than $D_{\max }-3 D$ before launching another robot. On the left, as the base decides to launch a new robot, robot a is too close to robot $\mathrm{b}$ and will withdraw. The next round is on the right: $\mathrm{b}$ is now too close to $\mathrm{c}$ and may withdraw, leaving only $\mathrm{c}$, which moves further away. As the new robot is launched, robot c should however be still visible from the base. The new robot $\circ$ was launched too late.

- be alive;

- have a smaller identifier that the observing robot;

- preferably have its light off, that is, if the target has its light on, then all robots within range also do;

- preferably select close robots, that is, if the target is in the pursuit zone and has its light on, then all robots within range are also in the pursuit zone.

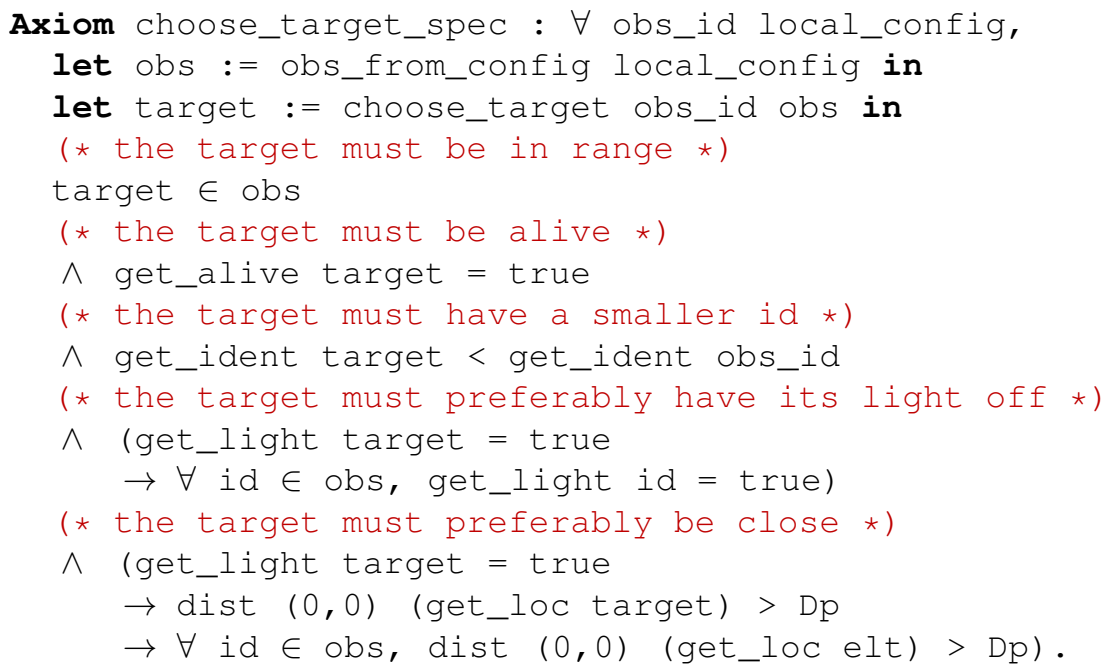

The second property specifies choose_new_pos: the new location is reachable with the speed of the observing robot (it is at most $D$ away from its current location) and not too far from its target (at most $D_{p}$ away from it, thus out of the pursuit zone).

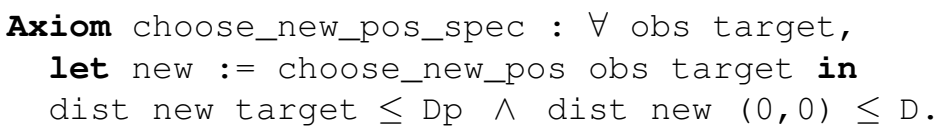

Finally, the specification of move_to is split in two depending on whether movement is possible or not. If it is possible, the chosen location is at least $2 D$ away from all other robots. 


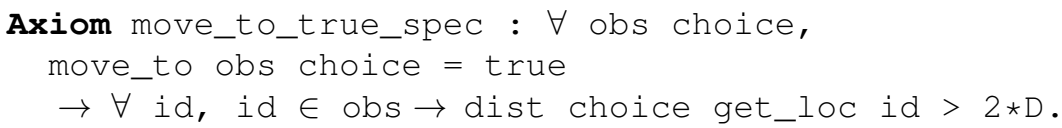

If movement is not possible, there is a robot with smaller identifier too close to the chosen location, that is, inside its danger zone (within a disc of radius $2 D$ ).

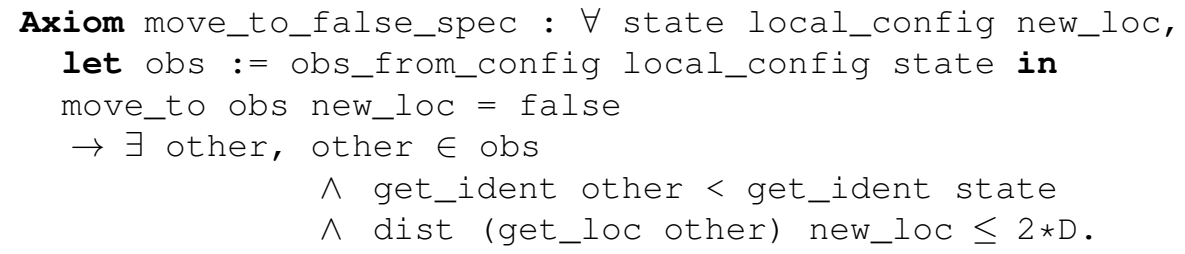

\section{Correctness proof of the family of solutions}

The proof amounts to ensuring that the property NoCollAndPath given in Section 4.1 is an invariant of the execution. This property combines no_collision_conf (the absence of collision) and path_conf (which entails the existence of a path from the base to the companion). We only sketch the proofs to convey their insight, for further detail we direct the interested reader to the COQ files.

The absence of collision can be directly proven to be an invariant. Assume that two robots that do not start at the same location collide after their move. Let $i d$ and $i d^{\prime}$ be the identifiers of the robots, with $i d<i d^{\prime}$. Since the robots are going to collide and none of them can travel more than $D$, they start at most $2 D$ apart. Since id observes a robot with lower identifier id in its danger zone, it does not move. Thus, to provoke a collision, id and $i \mathrm{~d}^{\prime}$ should start at most $D$ apart. In this case, $i \mathrm{~d}$ is actually in the collision zone of $i \mathrm{~d}^{\prime}$, so $i \mathrm{~d}^{\prime}$ withdraws and there is no collision.

Proving that the existence of a path is invariant is more subtle and we need to introduce three other properties that give more insight into the behaviour of the protocol:

- executed_means_light_on expressing that if a robot withdraws at the next round, then it has its light on (it is aware that it is in a potentially dangerous situation);

- executioner_means_light_off expressing that if a robot withdraws at the next round, then the robot causing this removal has its light off (ensuring that it cannot disappear too);

- exists_at_less_than_Dp expressing that if all robots of lower identifier in range of an alive robot $r$ have their light on, then one of them is not in the pursuit zone of $r$ (at most $D_{p}$ away from $r$ ).

These properties are proven by case analysis over two consecutive rounds, let us call them cf, cf'.

For the property executed_means_light_on, remark that if a robot $r$ alive in $c f$ withdraws in $c f^{\prime}$, it is either because, in $\mathrm{Cf}^{\prime}$, there is no robot in range or there is one too close (at most $D$ away). By contradiction, let us assume that $r$ withdraws in $\mathrm{Cf}^{\prime}$ while not deciding to turn its light on in $\mathrm{cf}$. Since the light is on when move_to returns false, we know this function returns true in $\mathrm{cf}$, and $r$ thus performs the move chosen by choose_new_pos between $\mathrm{cf}$ and $\mathrm{cf}^{\prime}$. Thus $r$ cannot lose contact with its target: by the specification of choose_new_pos $r$ moved at a distance no greater than $D_{p}$ to the cf-location of its target, that is at a distance no greater than $D_{P}+D=D_{\max }$ to the $\mathrm{cf}^{\prime}$-location of the target. Moreover, $r$ cannot withdraw due to another robot at a distance less than $D$ in $\mathrm{cf}^{\prime}$ : by the specification of choose_new_pos it moved at a distance more than $2 D$ apart from the cf-location of any other robot of lower identifier, that is more than $2 D-D=D$ apart from the $c f^{\prime}$-location of any other robot of lower identifier.

For the property executioner_means_light_off, note that by the definition of the abstract protocol, a moving robot always has its light off (by a simple case analysis on move_to).

By the property executed_means_light_on a robot $r$ alive in $\mathrm{cf}$ that withdraws in $\mathrm{Cf}^{\prime}$ has its light on and did not move between $\mathrm{cf}$ and $\mathrm{Cf}^{\prime}$. If $r$ stays alive in $\mathrm{cf}$ and withdraws in $\mathrm{Cf}^{\prime}$, then some other robot $r^{\prime}$ is at a distance from $r$ that is greater than $D$ in $\mathrm{cf}$ and at most $D$ in $\mathrm{Cf}^{\prime}$. Since $r$ does not move between the configurations, $r^{\prime}$ necessary does, and thus has its light of $\mathrm{f}$.

We show now that exists_at_less_than_Dp holds for $\mathrm{Cf}^{\prime}$. Let us consider an alive robot $r$ in $\mathrm{Cf}^{\prime}$ such that all robots in range have their light on. We want to prove that at least one of them is at most $D_{p}$ away. Note that as $r$ is alive in $\mathrm{Cf}^{\prime}$, there was a robot in range in $\mathrm{cf}$ and $r$ had a target $r^{\prime}$ in $\mathrm{cf}$. Since $r^{\prime}$ has its light on in $\mathrm{Cf} f^{\prime}$, 
it did not move between cf and $\mathrm{Cf}^{\prime}$. If $r^{\prime}$ was out of the pursuit zone of $r$ in cf (that is, at most $D_{p}$ away from $r$ ), we can conclude because $r$ either did not move or moved to a position not farther that $D_{p}$ away from $r^{\prime}$. If $r^{\prime}$ was inside the pursuit zone if $r$ in $\mathrm{cf}$, by the specification of choose_target, so were all robots in range of $r$. In particular, $r$ could move towards $r^{\prime}$ as no robot was inside its danger zone and since $r^{\prime}$ did not move, $r$ and $r^{\prime}$ are at most $D_{p}$ away in $\mathrm{cf}^{\prime}$.

Finally, let us turn to the proof that the property path_conf is an invariant. Let $r$ be a relay robot. We prove that it has a visible, active, launched neighbour with a smaller id. We consider several cases depending on the value of move_to and whether the target of $r$ withdraws.

If move_to is true and $r^{\prime}$ has its light off, then by property executioner_means_light_off $r^{\prime}$ cannot withdraw, and cannot get out of range of $r$ since $r$ moves closer to $r^{\prime}$.

If move_to is true and $r^{\prime}$ has its light on, the invariant exists_at_less_than_Dp and the specification of choose_target entail that all robots in range of $r$ have their light on and that $r^{\prime}$ is out of the pursuit zone. Hence, $r^{\prime}$ cannot withdraw since all robot close enough to eliminate it have their light on thus cannot cause it to withdraw (by executioner_means_light_off).

If move_to is false, then by move_to_false_spec, there is a robot with smaller id in range of $r$.

\section{Proof effort}

The proof effort for this work is decomposed in setup, specifications and actual proofs.

It consists of $\mathbf{5 2 0}$ lines of setup to instantiate the context (definition of robots state, observation, etc.), $\mathbf{5 4 0}$ lines of COQ of specifications to describe the problem.

The proof part is much more verbose, the table below show how many lines of proof tactics were used for the main invariants.

\begin{tabular}{lr}
\multicolumn{2}{c}{ Stability of main invariants } \\
\hline no_collision_conf & 1000 \\
executed_means_light_on & 2300 \\
executioner_means_light_off & 300 \\
exists_at_less_than_Dp & 1300 \\
path_conf & 50 \\
Auxiliary results & $\approx 5400$ \\
\hline Total for proofs & $\mathbf{1 0 5 0 0}$ \\
\hline
\end{tabular}

\subsection{Extracting a Sample Solution}

In the previous sections we designed a family of solutions defined by a template robogram function. The template robogram is parameterized by three auxiliary functions, each one being restricted by some axioms. Hence we can obtain a concrete solution by providing for each of the auxiliary functions a concrete definition that is consistent with the corresponding axioms.

It is again possible to develop such a concrete solution in our formal framework. Indeed, the PACTOLE library already provides some instances of concrete algorithms proven correct for other problems [4, 12]. However, if the axioms are simple enough one can also consider that most of the complex and error-prone reasoning on the model has been taken care of, and that a traditional pen-and-paper check provides a satisfying level of certainty.

In this section we exhibit a concrete solution, for which we check the axioms by hand. Please note that the selected solution has no particular property, and is not supposed to be more efficient, or better in any sense than the other members of our family of solutions. The concrete solution is chosen as one of the most straightforward validations of the axioms. In other words, the solution we present is a naive solution (or at least, it is naive once the axioms have been designed). Such a solution provides a sanity check of our axioms: it witnesses that our family of solutions is not empty.

\section{Concretisation of choose_target}

We take the point of view of a robot with identifier $n$, in a configuration satisfying the invariants of the algorithm. Define $V$ the subset of the observed robots that are

- alive

- at a distance at most $D_{\max }$ 
- with an identifier strictly smaller than $n$

If there is at least one robot in $V$ whose light is off, then select any robot in the subset $V_{\text {off }}$ of $V$ containing the robots with lights off. For instance, select the robot in $V_{\text {off }}$ with minimal identifier. If however all robots in $V$ have their lights on, then select any robot in the subset $V_{D p}$ of $V$ containing the robots at a distance at most $D_{p}$. For instance, select the robot in $V_{D p}$ with minimal identifier. Otherwise select any robot in $V$, for instance the robot in $V$ with minimal identifier.

We have to check that this function is defined and satisfies all the axioms. First note that, following the invariant path_conf, there is at least one robot that is alive, at a distance at most $D_{\max }$ of $n$ and with an identifier strictly smaller than $n$. Hence the set $V$ is not empty, and our function is defined. Second, the result of this function is a robot of $V$. As such it is visible, alive and and with an identifier strictly smaller than $n$ (three first parts of axiom choose_target_spec are satisfied). Third, the result has its light off as soon as there is at least one visible robot with its light off: fourth part of choose_target_spec is also satisfied. Finally, when there are only robots with their lights on, the result is a robot at a distance at most $D_{p}$ if there is one: the fifth and final part of axiom choose_target_spec is again satisfied. Hence our naive choose_target function satisfies all the relevant axioms.

\section{Concretisation of choose_new_pos}

We take the point of view of a robot with an already chosen target at distance at most $D_{\max }$. One can select the potential move of the robot as follows:

- if the target robot is at a distance greater than $D_{p}$, then choose a move of length $D$ toward the target;

- otherwise, choose a null move.

This tentative move aims at a position at distance at most $D$ from the starting position, and at most $D_{p}$ from the target. It thus complies with the axiom choose_new_pos_spec.

\section{Concretisation of move to}

We take the point of view of a robot with a potential move already selected, with some observed configuration. Validation of the potential move can be performed as follows:

- if there is any other robot at most $2 D$ away from the potential destination, then invalidate the potential move by returning false;

- otherwise, validate the potential move by returning true.

This function returns true only if there is no robot at a distance to the potential destination less or equal to $2 D$ : the axiom move_to_some_zone is satisfied. Conversely, the function returns false only if there is an observable other robot at a distance to the potential destination less or equal to $2 D$ : the axiom move_to_None is also satisfied.

Finally, the three parameter functions choose_target, choose_new_pos, and move_to all satisfy their respective axioms: they define a proper member of our family of solutions, which is not empty.

\section{Concluding remarks}

In this paper, we demonstrated by example how formal methods, and the PACTOLE framework in particular, can help mobile robotic swarm protocol designers to formally specify, design, and prove their algorithms are correct, balancing expressivity to tackle practically relevant problems, and formality to preserve the mathematical soundness of software developments.

Of course, proving correct algorithms for new problems is only the first step. A natural second step is to ensure the implementations of the algorithms maintain the relevant invariants when actually deployed on real devices. We leave this path for future research. 


\section{References}

[1] K. Altisen, P. Corbineau, and S. Devismes. A framework for certified self-stabilization. In E. Albert and I. Lanese, editors, Formal Techniques for Distributed Objects, Components, and Systems - 36th IFIP WG 6.1 International Conference, FORTE 2016, Held as Part of the 11th International Federated Conference on Distributed Computing Techniques, DisCoTec 2016, Heraklion, Crete, Greece, June 6-9, 2016, Proceedings, volume 9688 of Lecture Notes in Computer Science, pages 36-51. Springer-Verlag, 2016.

[2] C. Auger, Z. Bouzid, P. Courtieu, S. Tixeuil, and X. Urbain. Certified Impossibility Results for Byzantine-Tolerant Mobile Robots. In T. Higashino, Y. Katayama, T. Masuzawa, M. Potop-Butucaru, and M. Yamashita, editors, Stabilization, Safety, and Security of Distributed Systems - 15th International Symposium (SSS 2013), volume 8255 of Lecture Notes in Computer Science, pages 178-186, Osaka, Japan, Nov. 2013. Springer-Verlag.

[3] T. Balabonski, P. Courtieu, R. Pelle, L. Rieg, S. Tixeuil, and X. Urbain. Continuous vs. discrete asynchronous moves: A certified approach for mobile robots. In M. F. Atig and A. A. Schwarzmann, editors, Networked Systems - 7th International Conference, NETYS 2019, Marrakech, Morocco, June 19-21, 2019, Revised Selected Papers, volume 11704 of Lecture Notes in Computer Science, pages 93-109. Springer-Verlag, 2019.

[4] T. Balabonski, A. Delga, L. Rieg, S. Tixeuil, and X. Urbain. Synchronous gathering without multiplicity detection: A certified algorithm. Theory of Computing Systems, 2019. https://doi.org/10.1007/ s00224-017-9828-z.

[5] T. Balabonski, R. Pelle, L. Rieg, and S. Tixeuil. A foundational framework for certified impossibility results with mobile robots on graphs. In P. Bellavista and V. K. Garg, editors, Proceedings of the 19th International Conference on Distributed Computing and Networking, ICDCN 2018, Varanasi, India, January 4-7, 2018, pages 5:1-5:10. ACM, 2018.

[6] B. Bérard, P. Lafourcade, L. Millet, M. Potop-Butucaru, Y. Thierry-Mieg, and S. Tixeuil. Formal verification of mobile robot protocols. Distributed Computing, 29(6):459-487, 2016.

[7] M. Bezem, R. Bol, and J. F. Groote. Formalizing Process Algebraic Verifications in the Calculus of Constructions. Formal Aspects of Computing, 9:1-48, 1997.

[8] F. Bonnet, X. Défago, F. Petit, M. Potop-Butucaru, and S. Tixeuil. Discovering and assessing fine-grained metrics in robot networks protocols. In 33rd IEEE International Symposium on Reliable Distributed Systems Workshops, SRDS Workshops 2014, Nara, Japan, October 6-9, 2014, pages 50-59. IEEE, 2014.

[9] J. Castenow, P. Kling, T. Knollmann, and F. M. auf der Heide. A discrete and continuous study of the maxchain-formation problem: Slow down to speed up. In C. Scheideler and M. Spear, editors, SPAA '20: 32nd ACM Symposium on Parallelism in Algorithms and Architectures, Virtual Event, USA, July 15-17, 2020, pages 515-517. ACM, 2020.

[10] T. Coquand and C. Paulin-Mohring. Inductively Defined Types. In P. Martin-Löf and G. Mints, editors, International Conference on Computer Logic (Colog'88), volume 417 of Lecture Notes in Computer Science, pages 50-66. Springer-Verlag, 1990.

[11] P. Courtieu, L. Rieg, S. Tixeuil, and X. Urbain. Impossibility of Gathering, a Certification. Information Processing Letters, 115:447-452, 2015.

[12] P. Courtieu, L. Rieg, S. Tixeuil, and X. Urbain. Certified universal gathering algorithm in $\mathbb{R}^{2}$ for oblivious mobile robots. In C. Gavoille and D. Ilcinkas, editors, Distributed Computing - 30th International Symposium, (DISC 2016), volume 9888 of Lecture Notes in Computer Science, Paris, France, Sept. 2016. Springer-Verlag.

[13] D. Cousineau, D. Doligez, L. Lamport, S. Merz, D. Ricketts, and H. Vanzetto. TLA + Proofs. In D. Giannakopoulou and D. Méry, editors, FM, volume 7436 of Lecture Notes in Computer Science, pages 147-154, Paris, France, Aug. 2012. Springer-Verlag. 
[14] X. Défago, A. Heriban, S. Tixeuil, and K. Wada. Using model checking to formally verify rendezvous algorithms for robots with lights in euclidean space. In International Symposium on Reliable Distributed Systems, SRDS 2020, Shanghai, China, September 21-24, 2020, pages 113-122. IEEE, 2020.

[15] Y. Deng and J.-F. Monin. Verifying Self-stabilizing Population Protocols with Coq. In W.-N. Chin and S. Qin, editors, Third IEEE International Symposium on Theoretical Aspects of Software Engineering (TASE 2009), pages 201-208, Tianjin, China, July 2009. IEEE Computer Society.

[16] S. Devismes, A. Lamani, F. Petit, P. Raymond, and S. Tixeuil. Optimal Grid Exploration by Asynchronous Oblivious Robots. In A. W. Richa and C. Scheideler, editors, Stabilization, Safety, and Security of Distributed Systems - 14th International Symposium (SSS 2012), volume 7596 of Lecture Notes in Computer Science, pages 64-76, Toronto, Canada, Oct. 2012. Springer-Verlag.

[17] H. T. T. Doan, F. Bonnet, and K. Ogata. Model checking of a mobile robots perpetual exploration algorithm. In S. Liu, Z. Duan, C. Tian, and F. Nagoya, editors, Structured Object-Oriented Formal Language and Method - 6th International Workshop, SOFL+MSVL 2016, Tokyo, Japan, November 15, 2016, Revised Selected Papers, volume 10189 of Lecture Notes in Computer Science, pages 201-219, 2016.

[18] H. T. T. Doan, F. Bonnet, and K. Ogata. Model checking of robot gathering. In J. Aspnes, A. Bessani, P. Felber, and J. Leitão, editors, 21 st International Conference on Principles of Distributed Systems, OPODIS 2017, Lisbon, Portugal, December 18-20, 2017, volume 95 of LIPIcs, pages 12:1-12:16. Schloss Dagstuhl Leibniz-Zentrum für Informatik, 2017.

[19] P. Flocchini, G. Prencipe, N. Santoro, and P. Widmayer. Gathering of asynchronous robots with limited visibility. Theor. Comput. Sci., 337(1-3):147-168, 2005.

[20] W. Fokkink. Modelling Distributed Systems. EATCS Texts in Theoretical Computer Science. SpringerVerlag, 2007.

[21] N. Gaspar, L. Henrio, and E. Madelaine. Bringing coq into the world of gcm distributed applications. pages 643-662, 2014.

[22] P. Kling and F. M. auf der Heide. Continuous protocols for swarm robotics. In P. Flocchini, G. Prencipe, and N. Santoro, editors, Distributed Computing by Mobile Entities, Current Research in Moving and Computing, volume 11340 of Lecture Notes in Computer Science, pages 317-334. Springer, 2019.

[23] P. Küfner, U. Nestmann, and C. Rickmann. Formal Verification of Distributed Algorithms - From Pseudo Code to Checked Proofs. In J. C. M. Baeten, T. Ball, and F. S. de Boer, editors, IFIP TCS, volume 7604 of Lecture Notes in Computer Science, pages 209-224, Amsterdam, The Netherlands, Sept. 2012. SpringerVerlag.

[24] L. Lamport. The temporal logic of actions. ACM Trans. Program. Lang. Syst., 16(3):872-923, May 1994.

[25] L. Millet, M. Potop-Butucaru, N. Sznajder, and S. Tixeuil. On the synthesis of mobile robots algorithms: The case of ring gathering. In P. Felber and V. K. Garg, editors, Stabilization, Safety, and Security of Distributed Systems - 16th International Symposium, (SSS 2014), volume 8756 of Lecture Notes in Computer Science, pages 237-251, Paderborn, Germany, Sept. 2014. Springer-Verlag.

[26] L. Reynaud and I. G. Lassous. Design of a force-based controlled mobility on aerial vehicles for pest management. Ad-Hoc Networks, 53:41-52, 2016.

[27] A. Sangnier, N. Sznajder, M. Potop-Butucaru, and S. Tixeuil. Parameterized verification of algorithms for oblivious robots on a ring. Formal Methods Syst. Des., 56(1):55-89, 2020.

[28] I. Suzuki and M. Yamashita. Distributed Anonymous Mobile Robots: Formation of Geometric Patterns. SIAM Journal of Computing, 28(4):1347-1363, 1999. 